\title{
»Auf das Verhalten anderer bezogen und daran in seinem Ablauf orientiert«
}

\author{
Bodo de Vries
}

\begin{abstract}
Sozialwirtschaftliche Unternehmen freier Träger müssen sich durch ein besonderes »soziales Handeln "auszeichnen. Dieses Handeln kann im Sinne von Max Weber verstanden werden als "auf das Verhalten anderer bezogen (...) und daran in seinem Ablauf orientiert «. Diese besondere Orientierung setzt das Sozialunternehmen vielfältigen wirtschaftlichen, administrativen, rechtlichen und werteorientierten Spannungen aus.
\end{abstract}

Sozialunternehmen sind vielfältigen Anforderungen ausgesetzt und werden in der Fachliteratur selbst mit umfassenden Vorgaben und Definitionen bedacht. In diesem Beitrag werden Sozialunternehmen als Non-Profit-Organisation verstanden, die sich dadurch auszeichnen, dass sie ihr Handeln auf eine spezifische Weise ausgerichtet haben. Diese entsteht durch eine besondere Grundorientierung, die in Anlehnung von Max Weber das »soziale Handeln« der Sozialunternehmen als Apriori berücksichtigt.

Das Handeln von Sozialunternehmen ist »seinem von dem oder den Handelnden gemeinten Sinn nach auf das Verhalten anderer bezogen (...) und daran in seinem Ablauf orientiert (...)«. (1) Diese besondere Orientierung »auf das Verhalten anderer « bedeutet für die Sozialunternehmen umfassende Spannungsfelder von wirtschaftlichen, administrativen, rechtlichen oder auch werteorientierten Handlungssphären, mit denen die Bewohner von Einrichtungen, Kunden, Patienten u. a. (2) das Unternehmen identifizieren.

Diese besondere Ausrichtung auf das Soziale Handeln kann als Vorgabe für Brauchtums- und Freizeitvereine gelten, die aus Kultur- oder Sportgruppen hervorgegangen sind, oder auf die Sozialunternehmen der Wohlfahrtspflege bezogen werden, die auf konfessionelle Gründungskontexte und soziale Problemlagen gründen. Häufig lässt sich diese Grundorientierung der Sozialunternehmen nicht vollständig im Alltagshandeln identifizieren. Hierfür existieren verschiedene Ursachen und Begründungszusammenhänge:

- Pluralisierende Anforderungen an das Alltagshandeln überdecken die Grundorientierung.

- Erlebte Widersprüche in Organisationsvorgaben werden von Mitarbeitern und Kunden reklamiert.

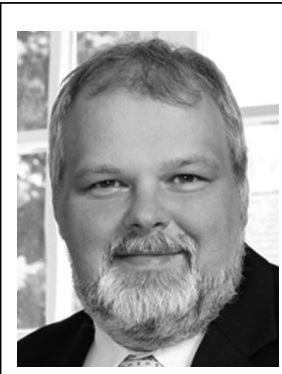

Dr. Bodo de Vries (44) studierte Sozialwissenschaft an der Bergischen Universität Wuppertal und der Alliance Francaise in Paris. Nach einer Zeit als wissenschaftlicher Mitarbeiter an der Bergischen Universität Wuppertal und seiner Promotion baute er die Europäische Senioren-Akademie in

Ahaus auf, die er dann zehn Jahre leitete. Seit 2006 ist er Geschäftsführer des Geschäftsbereichs Soziale Arbeit und Gesundheit im Ev. Johanneswerk und seit letztem Jahr in dessen Vorstand.

Internet http://www.johanneswerk.de

- Die Handlungsbereiche des Sozialunternehmens (z. B Behindertenhilfe, Altenhilfe, Erziehungshilfe u. a. m.) haben pluralisierende Milieus hervorgebracht, deren Koexistenz im Unternehmen und Alltagshandeln Widersprüche produziert.

- Die Führung von Sozialunternehmen ist nicht in der Lage, die erlebten Spannungsfelder unterschiedlicher Anforderungen so zu kommunizieren, dass die Grundorientierung nachvollziehbar und glaubwürdig erkennbar bleibt.

\section{Die Grundorientierung in der Wohlfahrtspflege}

In der Wohlfahrtspflege wird die Grundorientierung häufig auch aus Gründungskontexten und Leitmotiven der Vergangenheit abgeleitet, die als soziale Bewegung mit verbindenden Ideen oder konfessionellen Weltorientierungen den Ursprung des Sozialunternehmens ausmachen. Sie werden durch Rituale und Organisationstraditionen gefüllt. Dominieren diese Rituale und Organisationstraditionen und kommt es parallel durch weitere Handlungsorientierungen zu keiner oder einer reduzierten Ansprache lebensweltlicher Bedingungen und Problemlagen von Kunden, entsteht zwangsläufig eine Schieflage oder Einseitigkeit, die das Sozialunternehmen 
als Sozialunternehmen im hier verstandenen Sinne angreift und reduziert.

Diese Bewertung leitet sich aus Webers Vorgabe des sozialen Handelns unmittelbar ab: Die Einseitigkeit bedient die Handlungsorientierungen der Kunden so unvollständig, dass man den Unternehmen ein Handeln absprechen muss, das tatsächlich auf »das Verhalten anderer bezogen ist und sich in seinem Ablauf an diesem orientiert«. Rationale, affektuelle und wertrationale Handlungskategorien, welche die Lebenswelten der Kunden und Mitarbeiter ansprechen, aufnehmen und determinieren, drohen unberücksichtigt zu bleiben. Kommt es hier zu keiner Adaption und Akzeptanz an die Grundorientierung verliert das "Sozialunternehmen « im hier verstandenen Sinne des Wortes seine Existenzberechtigung. Die Sozialunternehmen sind nicht mehr »sozial« und werden $\mathrm{zu}$ anderen Formen von Unternehmungen.

Vor diesem Hintergrund ist eine Unternehmensführung, die sich dieser theoretischen Zusammenhänge bewusst ist und die die notwendigen sozialen, personalen, fachlichen und methodischen Kompetenzen entfaltet, um diese Grundorientierung mit Leben zu füllen, von existenzieller Bedeutung für das Sozialunternehmen.

\section{Beispiel stationäre Altenpflege}

In diesem Zusammenhang könnte beispielsweise die Neigung, an der stationären Versorgung alter Menschen in Einrichtungen einseitig festzuhalten, zu einer umfassenden Problemstellung werden. Es lässt sich zwar für viele Sozialunternehmen der Wohlfahrtspflege eine über Jahrzehnte darstellbare Tradition belegen, die mit einer christlich motivierten Grundorientierung und imponierenden Versorgungsleistungen belegt werden kann.

Wenn die gesellschaftliche Entwicklung und damit die Kunden und Mitarbeiter zu alternativen Versorgungsformen motivieren, also zu einer stationären Versorgungsqualität, die jenseits von Einrichtungen und Anstalten liegt; wenn veränderte Werte, Wünsche und Ängste von Kunden zunehmend die Entwicklung einer Versorgungsqualität erfordern, die nicht nur reichen Pflegebedürftigen den Verbleib in den eigenen vier Wänden ermöglichen, sondern hier ein gesamtgesellschaftliches Angebot fordern, verlieren Sozialunternehmen im oben verstandenen Sinn des Wortes Ihre Existenzberechtigung, wenn Sie einseitig und ausschließlich den Ausbau des stationären Regelsystems der Altenpflege in Einrichtungen und Anstalten propagieren. Sozialunternehmen müssen deshalb eher als der Staat und der rein wirtschaftlich orientierte Markt den gesellschaftlichen Bedarf und die Bedürfnislagen der Kunden erkennen und aufgreifen und auf dieser Grundlage alternative Versorgungssettings entwickeln oder den Markt und den Staat hierzu motivieren oder auffordern.

Vor diesem Hintergrund wird deutlich, warum die umfassende Expansion stationärer Einrichtungen in Deutschland sehr wesentlich nicht von Sozialunternehmen vorangetrieben werden kann und darf. Demografisch, pflegerisch, epidemiologisch wird hier an den Bedarfs- und Bedürfnislagen der Kunden »vorbeiproduziert « und geplant. Der sozialwissenschaftlich längst und umfassend belegte Wunsch pflegebedürftiger alter Menschen, in den eigenen vier Wänden eine bessere und nachhaltige Versorgungsqualität zu erhalten, wird aus wirtschaftlichen Motiven weitgehend ignoriert. (3) Übervorsichtige gesetzliche Wege, dem Postulat »ambulant vor stationär « tatsächlich neue Wege zu ebnen, um alternative Entwicklungspotenziale zu fördern, lassen sich wenig erkennen und unterscheiden sich sogar von Bundesland zu Bundesland. Dies belegen die länderspezifischen Formulierungen der Heimgesetzgebung.

Auch leisten Sozialunternehmen, die aus der institutionellen Nähe von Staat und Kommune hervorgegangen sind, in diesem Bereich wenig Entwicklungsarbeit. Politische Zielsetzungen dieser Sozialunternehmen resultieren deutlich aus dem politischen Nahbereich von staatlicher oder kommunaler Verwaltung. Wesentliche Zielsetzung ist eine einseitige Wirkungsoptimierung und ein Kostenbewusstsein der Aufgabenerfüllung der stationären Versorgung. Damit fallen diese Unternehmen weitgehend für die Weiterentwicklung alternativer Versorgungssettings, die veränderte gesellschaftliche Bedarfs- und Bedürfnislagen aufgreifen, aus. Zauner attestiert dieser Folgebereitschaft des staatlich-administrativen Agierens dieser NonProfit-Unternehmen eine weitgehende »gesellschaftliche Ignoranz«. (4) Einer Bewertung der man sich nicht anschließen muss, wenn man im hier dargestellten theoretischen Kontext bleibt. Die Handlungsorientierungen und Kategorien des sozialen Handelns Webers lassen sich hier Idealtypen verstehen, die es in der definitorischen Reinheit in der Wirklichkeit nicht geben kann, die also zwangsläufig von diesen abweicht.

\section{Sozialunternehmen im gesellschaftlichen Wandel}

Aus diesen Überlegungen leitet sich die These ab, dass Sozialunternehmen, die sich an den gesellschaftlichen Anforderungen, also den Bedarfs- und Bedürfnislagen ih-

\section{"Die Lebenswelt von Kunden und Mitarbeitenden muss ständig im Blick bleiben «}

rer Kunden einerseits orientieren und sich andererseits den Anforderungen, die sich aus staatlichen Vorgaben und dem Marktgeschehen in ihrem Handlungsbereich oder Branche stellen, die Aufgabe haben, sich dem Wandel gesellschaftlicher Anforderungen ihrer Versorgungsleistungen zu stellen. Wie kann das funktionieren? Welche Rahmenbedingungen bedarf es hierfür im Sozialunternehmen?

Zunächst bedarf es sicherlich der Einsicht, dass sowohl eine einseitige wirtschaftliche Orientierung, wie auch eine einseitige staatlich-administrative Folgebereitschaft des Sozialunternehmens ins »Soziografische Niemandsland « führt und durch das unreflektierte Agieren zwischen Staat und Markt mittelfristig die Orientierung an 
gesellschaftlichen Bedarfs- und Bedürfnislagen aufgegeben wird. Natürlich können neue stationäre Einrichtungen der Altenpflege kurz- und mittelfristig wirtschaftlich sein und die Marktposition eines Unternehmens verbessern. Auch kann das einseitige Verharren an traditionellen Vorgaben des stationären Regelsystems den staatlichkommunalen Bedarfslagen zuarbeiten, die entsprechend der Stabilisierung des Aufgabenvollzugs der stationären Altenpflege eine befriedigende administrative Aufgabenerfüllung bedeuten.

Alternative Versorgungssettings auf der Grundlage veränderter Bedarfs- und Bedürfnislagen älterer Menschen entstehen jedoch in und durch Sozialunternehmen, die sich ihrer Verankerung und Entstehung aus der sozialen Bewegung und ihrer Grundorientierung bewusst sind und diese als Gestaltungsquelle ihrer Arbeit und ihrer Positionierung zwischen Staat und Markt nutzen. Die Basisnähe zu karitativen Vereinigungen, Solidaritätsgruppen, politischen Vereinigungen sowie das weite Spektrum des kulturellen und freizeitorientierten Vereinswesens ermöglichen Sozialunternehmen, die die Vorgaben von Markt und Staat nutzen oder diese gestalten, eine zivilgesellschaftliche Positionierung, die ihre Sonderstellung als Unternehmen im Wirtschaftsgefüge rechtfertigt und garantieren muss.

\section{Sozialunternehmen und der demografische Wandel}

Bezogen auf das bereits bemühte Beispiel der stationären Versorgung lässt sich darstellen, wie Staat, Markt und Sozialunternehmen agieren und ihren besonderen Rollen gerecht werden. Für den Markt und den Staat sind die Anforderungen, die aus dem demografischen Wandel abgeleitet werden, gesamtgesellschaftliche Anpassungsleistungen. Der Markt hofft auf eine parallele Anpassung stationärer Versorgungsstrukturen an einen steigenden Bedarf der Versorgung hilfs- und pflegebedürftiger alter Menschen. Der Staat deutet den steigenden Anteil alter und pflegebedürftiger Menschen als Anforderung, Anpassungsleistungen in bestehenden Sicherungssystemen der Kranken- und Pflegeversicherung vorzunehmen. Kurzund mittelfristig könnte sich jedes Sozialunternehmen an diesen Vorgaben adaptieren und sich erfolgreich gesellschaftlich, marktpolitisch und im kommunalen Gemeinwesen behaupten.

Die Einseitigkeit dieser Orientierungen wird erst dann offenbar, wenn sich das Sozialunternehmen auf seine Grundorientierung besinnt und die Veränderungen als das wahrnimmt, was sie sind: Der demografische Wandel ist für sich genommen nichts anderes als eine empirisch belegbare Veränderung und Beschreibung der Größen von Altersklassen oder Alterskohorten einer Gesellschaft. Demografieforschung bewertet nicht, sondern beschreibt.

Das eigentliche Phänomen, das für das Sozialunternehmen von Bedeutung ist, ist der gesellschaftliche Wandel, der aus dem demografischen Wandel abgeleitet werden muss und die Beziehungsgeflechte transformiert, in denen beispielsweise ältere Menschen eingebunden sind: Wer keine Kinder oder weniger Kinder hat, der wird auch keine oder weniger Enkel haben. In einer Gesellschaft, in der die Altersklasse Älterer wächst, sind diese zunehmend auf intragenerative Formen der Beziehungsgestaltung angewiesen. Die Alten werden zunehmend unter sich bleiben.

Diese und weitere Konsequenzen für die Beziehungsgeflechte und sozialen Netzwerke sind für das Sozialunternehmen von existenzieller Bedeutung und machen ein grundsätzliches Überdenken bestehender Versorgungssysteme notwendig. Die Trägheit staatlich-administrativer Systeme und die wirtschaftliche Marktorientierung sind ungeeignet, den Wandel von daraus resultierenden Bedarfs- und Bedürfnislagen älterer Menschen eine gesellschaftliche Stimme zu verleihen und entsprechend zu gestalten.

Sicherlich ist bekannt, dass der Wohn- und Sterbeort der stationären Altenpflege für einen großen Teil älterer Menschen an Akzeptanz im Verhältnis zu früheren Generationen verloren hat. Die Finanzierung der Versorgung von heutigen circa 30 Prozent der Pflegebedürftigen in stationären Einrichtungen wird sich in den aktuellen Regelsystemen auch nicht mit der heutigen Versorgungsqualität für zukünftige Generationen darstellen lassen. Diese Rahmenvorgaben sind jedoch keine hinlängliche Grundlage dafür, dass Marktentwicklungen und staatliche Regelsysteme sich in einer hinreichenden Qualität den gesellschaftlichen Wandel und veränderten Bedarfs- und Bedürfnislagen stellen. Sozialunternehmen, die sich der oben dargestellten Grundorientierung bewusst bleiben, haben hier die besondere Chance durch zivilgesellschaftliche Potenziale, durch partizipative Versorgungskonzepte Alternativen zu gestalten.

Die Transformation einer stationären Versorgungsqualität in das ambulante Regelsystem und in das Wohnquartier könnte im Kontext der gesellschaftlichen Bedarfs- und Bedürfnislage zu einer Initiative von Sozialunternehmen werden. Einige Sozialunternehmen in Deutschland arbeiten hier bereits an der Entwicklung veränderter Versorgungskonzepte, die mit Wohncafés, Pflegestützpunkten, einer moderierten Kultur- und Freizeitarbeit zivilgesellschaftliche Impulse setzen und nutzen, um Alternativen zur stationären Versorgung alter Menschen zu schaffen, die neben Alten- und Pflegeheimen die Versorgungssettings in Deutschland pluralisieren werden. Die quartiersnahe Versorgung im Ev. Johanneswerk versteht sich in diesem Zusammenhang als Mitgestalter dieser Veränderungen.

\section{Anmerkungen}

(1) Weber, Max; Wirtschaft und Gesellschaft; § 1.

(2) Im Folgenden werden die Nutzer von Sozialunternehmen unter dem Begriff »Kunden« subsumiert, ohne hiermit eine Positionierung zu den vielschichtigen Deutungen dieses Begriffes vorgenommen zu haben.

(3) Vgl.: Institut für Demoskopie Allenbach; Altwerden im 21. Jahrhundert; 2001.

(4) Zauner, A.; Über Solidarität zu Wissen. Ein systemtheoretischer Zugang zu Nonprofit Organisationen; in: Badelt, Ch, u. a.; Handbuch der Nonprofit Organsiation; Stuttgart 2007; S. 148. 